\author{
Stability analysis of sdO equilibrium models \\ C. Rodríguez-López, ${ }^{1,2}$ R. Garrido, ${ }^{1}$ A. Moya, ${ }^{1}$ J. MacDonald, ${ }^{3}$ A. Ulla ${ }^{2}$ \\ ${ }^{1}$ Instituto de Astrofísica de Andalucía-CSIC, E 18008 Granada, España \\ 2 Universidad de Vigo, E 36200 Vigo, España \\ ${ }^{3}$ University of Delaware, DE 19176 Newark, USA
}

\begin{abstract}
We present fully nonadiabatic calculations describing the driving of pulsations in equilibrium models of sdO stars. The first pulsating sdO, SDSS J160043.6+074802.9 has been recently discovered showing short period oscillations suggesting $\mathrm{p}$ modes.
\end{abstract}

\title{
Stability analysis
}

Hot subdwarf $\mathrm{O}$ stars (sdOs) are blue subluminous objects in a stage immediately prior to the white dwarf phase. With the final aim of using the tools of asteroseismology to unravel the sdO's evolutionary state, we undertook a theoretical stability analysis to explore their feasibility as pulsators.

The evolution code JMSTAR (Lawlor \& MacDonald 2006) was used to calculate a total of $16 \mathrm{sdO}$ equilibrium models corresponding to different full evolutionary sequences. We used the nonadiabatic code of oscillations GRACO (GRAnada COde, Moya et al. 2004) to perform the nonadiabatic analysis (Rodríguez-López 2007). Out of the 16 models, one ( $T_{\text {eff }}=45000 \mathrm{~K}$, $\log g=4.2, Z=0.14$ ) was found to drive unstable modes. We plot below the normalized growth rate, $\eta$, vs. frequency as an indicator of the stability $(\eta<0)$ or instability $(\eta>0)$ of the mode; and the derivative of the work integral and opacity vs. $\log q\left(=1-M_{r} / M\right)$. A negative (positive) value of $\mathrm{d} W / \mathrm{d} \log q$ at a given location in the model indicates that this region contributes locally to driving (damping) of the mode.

We found excited modes, for $\ell=2$, within the frequency ranges: $0.29 \leq \nu \leq 0.32$ and $0.38 \leq \nu \leq 0.42 \mathrm{mHz}$. The excited modes fall within a wider range favoured for instability $0.2 \leq \nu \leq 1.5 \mathrm{mHz}$. Meanwhile, modes with frequencies $\nu \geq 2 \mathrm{mHz}$ were found highly stable with values of $\eta=-1$.

Figure 1 (bottom left) plots the g190 mode with $\nu=0.31 \mathrm{mHz}$ and $\eta=0.17$ as representative of the two zones of instability. There is a wide driving zone at the location of the $Z$-bump. Hence, the instability is explained by the classical $\kappa$-mechanism associated with partial ionization of heavy elements in the envelope of the star, the same mechanism that drives oscillations in sdB pulsators. In this case, the excited modes correspond to high radial order g modes.

Figure 1 (bottom right) plots the g177 mode with $\nu=0.33 \mathrm{mHz}$ and $\eta=-0.95$ representative of the stability zones. The stability is due to the near extinction of the previous driving region and all the significant energy contributing to damp the modes. The development and extinction of a region contributing to driving (with its maximum taking place at the location of the Z-bump) at the expense of a damping region is responsible for the oscillatory profile of the growth rate.

This model does not excite $\mathrm{p}$ modes which are the only ones found to date in the discovered sdO pulsator (Woudt et al. 2006). Some of our models, however, present a tendency to drive low radial order p modes (Rodríguez-López et al. 2006, Rodríguez-López 2007). 

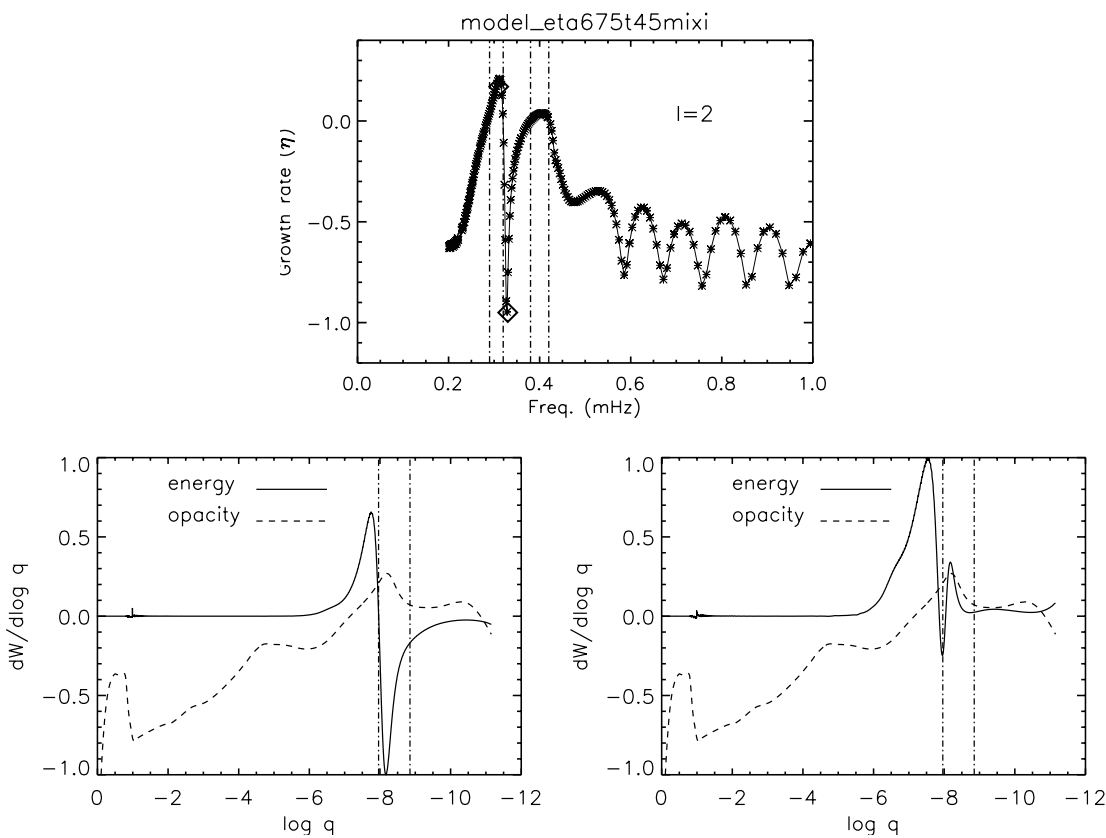

Figure 1: Top: Growth rate parameter vs. frequency in the region of interest. The dashed-dotted lines represent the two regions of unstable modes and the diamonds mark the modes plotted beside. Bottom: Energy and opacity for the g190 and g177 modes, respectively. The vertical dashed-dotted line depicts the convection zone. Both plots have been scaled to arbitrary units.

Acknowledgments. This work was supported by the Spanish Ministerio de Ciencie y Tecnología under project ESP2004-03855-c03-01.

\section{References}

Lawlor T. M., MacDonald J., 2006, MNRAS, 371, 263

Moya A., Garrido R., Dupret M.-A., 2004, A\&A 404, 1081

Rodríguez-López C.. 2007, Ph.D. Thesis, Universidad de Vigo

Rodríguez-López C., Moya A., Garrido R., et al., 2006, Baltic Astronomy 15, 313

Woudt P. A., Kilkenny D., Zietsman E., et al., 2006, MNRAS, 371, 1497 